\title{
Evaluation of a 12-mm diameter covered self-expandable end bare metal stent for malignant biliary obstruction
}

\section{(ㄷ)(1) $\odot$}

\author{
Authors \\ Kazunori Nakaoka, Senju Hashimoto, Naoto Kawabe, Takuji Nakano, Toshiki Kan, Masashi Ohki, Yuka Ochi, Tomoki \\ Takamura, Takamitsu Kurashita, Sayuri Nomura, Keishi Koyama, Aiko Fukui, Kentaro Yoshioka
}

Institution

Department of Liver, Biliary Tract and Pancreas Diseases, Fujita Health University School of Medicine, Aichi, Japan

submitted 4.12 .2017

accepted after revision 27.4.2018

Bibliography

DOI https://doi.org/10.1055/a-0627-7078 |

Endoscopy International Open 2018; 06: E1164-E1170

(c) Georg Thieme Verlag KG Stuttgart · New York

ISSN 2364-3722

Corresponding author

Senju Hashimoto, MD, Department of Liver, Biliary Tract

and Pancreas Diseases, Fujita Health University, 1-98

Dengakugakubo, Kutsukakecho, Toyoake, Aichi 470-1192,

Japan

Fax: +81-562-93-8601

hsenju@fujita-hu.ac.jp

\section{ABSTRACT}

Background and study aims Biliary metallic stents are used to drain unresectable malignant distal biliary obstructions. This study aimed to evaluate the efficacy of a novel 12-mm-diameter covered, self-expandable end bare metal stent (12-mm CSEEMS).
Patients and methods We evaluated 99 patients with unresectable malignant distal biliary obstructions treated with covered biliary metallic stents. Of the 99 patients, 33 underwent 12-mm CSEEMS placement between June 2015 and April 2017 (12-mm-CSEEMS group) and 66 underwent 10-mm fully-covered self-expandable metal stent (FCSEMS) placement between January 2010 and July 2015 (10-mmFCSEMS group). The overall survival (OS), the recurrent biliary obstruction (RBO), cause of RBO, time to RBO (TRBO) and adverse events in 12-mm-CSEEMS group and 10-mmFCSEMS group were evaluated retrospectively.

Results The OS tended to be longer in the 12-mm-CSEEMS group (log rank, $P=0.081$ ) and TRBO was significantly longer in the 12 -mm-CSEEMS group (log rank, $P=0.001$ ) than in the 10-mm-FCSEMS group. Both univariate (HR, 0.449; $95 \%$ $\mathrm{Cl}, 0.27967-0.72215 ; P=0.001)$ and multivariate (HR, 0.458 ; $95 \% \mathrm{Cl}, 0.28395-0.73744 ; P=0.001$ ) Cox hazard analysis found that risk of RBO was significantly lower in 12$\mathrm{mm}$ CSEEMS than in 10-mm FCSEMS. There were no significant differences between the 12-mm-CSEEMS group and $10-\mathrm{mm}$-FCSEMS group regarding the cause of RBO and adverse events.

Conclusions The 12-mm CSEEMS showed a low risk of RBO compared with 10-mm FCSEMS and was considered to be effective and safe for draining unresectable malignant distal biliary obstruction.

\section{Introduction}

Endoscopic drainage of malignant biliary obstruction using selfexpandable metal stents (SEMSs) is a widely used standard procedure to treat obstructive jaundice which enables chemotherapy and improves patients' symptoms [1-5]. Covered SEMSs (CSEMSs) may prevent tumor ingrowth more effectively than uncovered SEMSs (USEMSs) [6]. In patients with malignant biliary obstruction, Isayama et al. found that the time to recurrent biliary obstruction (RBO) was longer with CSEMS than with USEMS [5]. In patients with biliary obstruction caused by pan- creatic carcinoma, Kitano et al. reported that duration of patency was longer with CSEMS than with USEMS [7].

However, several meta-analyses reported that CSEMS has a higher risk of migration than USEMS, despite prevention of ingrowth $[1-3,8]$. Mukai et al. developed a 12-mm-diameter fully-covered self-expandable metal stent (FCSEMS) to prevent RBO, but it resulted in several cases of migration [9]. Therefore, we evaluated the efficiency of a 12-mm-diameter covered selfexpandable-end, bare metal stent (CSEEMS) in patients with malignant distal biliary obstruction for preventing RBO. 


\section{Patients and methods}

We retrospectively evaluated 99 patients with unresectable malignant distal biliary obstructions treated with covered biliary metallic stents at Fujita Health University Hospital. Of the 99 patients, 33 underwent placement of 12-mm-diameter CSEEMS (Tae Woong Medical, Seoul, Korea) between June 2015 and April 2017 (12-mm-CSEEMS group) (ฉ Fig. 1) and 66 underwent 10-mm-diameter FCSEMS (Wallflex biliary RX stent, Boston Scientific, Natick, Massachusetts) placement between January 2010 and July 2015 (10-mm-FCSEMS group).

The endpoint of this study was RBO with SEMS, or patients' death, whichever was earlier. The patients survived during the observation period were considered as censored cases.

Before inserting these metal stents, carcinoma was diagnosed by cytology, biopsy, or endoscopic ultrasound-guided fine-needle aspiration. If diagnosis by tissue biopsy or cytology was not possible, enhanced computed tomography (CT) or magnetic resonance imaging was used. We initially performed drainage using a plastic stent and then switched the plastic stent with a 12-mm CSEEMS or 10-mm FCSEMS after confirming that there was no indication for surgery and that the patients had good life expectancy. Thereafter, the patients were treated with chemotherapy or optimal supportive care.

\section{Eligibility criteria}

Patients who were age $\geq 20$ years and those with a life expectancy $\geq 3$ month, an Eastern Cooperative Oncology Group Performance Status (ECOG-PS) < 4 and diagnosed with distal biliary obstruction caused by an unresectable malignancy were included. Patients with ECOG-PS $\geq 4$, massive ascites, an intestinal obstruction distal to the ampulla, and prior biliary SEMS placement and those who were unable to give informed consent for SEMS replacements were excluded.

\section{Ethical affairs}

The study protocol was approved by the Institutional Review Board of Fujita Health University Hospital (HM16-059) and was carried out following the ethical principles of the Declaration of Helsinki.

\section{SEMS used in the study}

A 12-mm CSEEMS is made of nitinol wire and covered with a silicone membrane, with the proximal $10 \mathrm{~mm}$ uncovered and distal 5-mm flared ends designed to prevent migration. The area of $12-\mathrm{mm}$ CSEEMS was 1.44 -fold larger than that of $10-\mathrm{mm}$ FCSEMS. A 12-mm CSEEMS is available in lengths of 6,7 , and $8 \mathrm{~cm}$ and is equipped with a 9-Fr standard delivery device. For 12-mm CSEEMS, the axial force (AF) at a 20-mm distance from the bending point was $0.29 \mathrm{~N}$ and the radial force (RF) measured at a $4-\mathrm{mm}$ diameter was $4.5 \mathrm{~N}$, as previously described [10]. A 12-mm CSEEMS was newly manufactured just before this study ( $\triangleright$ Fig.1).

A $10-\mathrm{mm}$ FCSEMS is made of nitinol wire and covered with a silicone membrane, with both ends flared. A 10-mm FCSEMS is available in lengths of 4,6 , and $8 \mathrm{~cm}$ and equipped with an 8.5Fr standard delivery device. The AF of this stent at a $20-\mathrm{mm}$ dis-

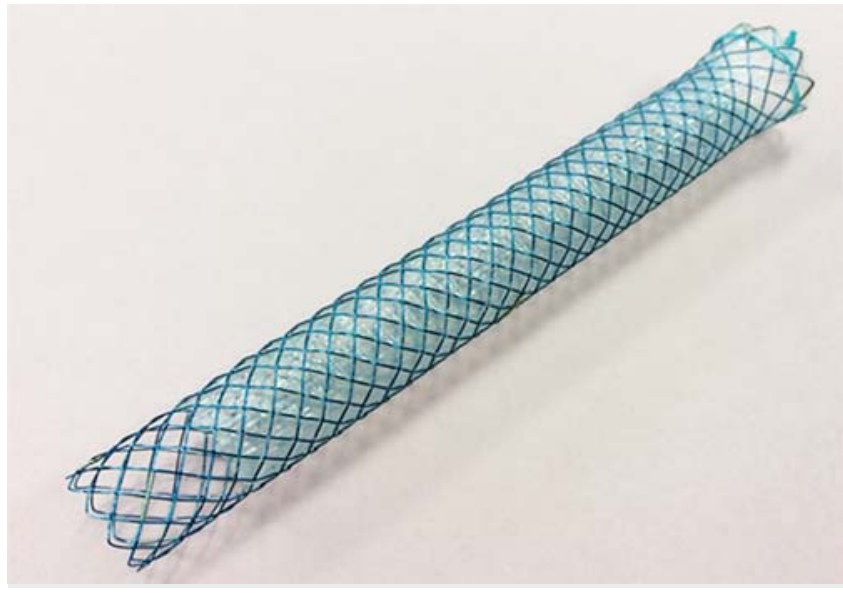

- Fig. 1 A 12-mm-diameter covered self-expandable-end bare metal stent (CSEEMS). This SEMS is made of nitinol wire and covered with a silicone membrane. The proximal $10 \mathrm{~mm}$ is uncovered, and $5 \mathrm{~mm}$ of the distal end is flared to prevent migration. The area of 12 -mm CSEEMS is 1.44-fold larger than that of the 10-mm SEMS.

tance from the bending point was $0.65 \mathrm{~N}$, and RF measured at a 4-mm diameter was $4.7 \mathrm{~N}$.

\section{Procedures}

SEMS was inserted during endoscopic retrograde cholangiopancreatography (ERCP) by two experienced investigators using a standard duodenoscope (TJF-260V; Olympus, Tokyo, Japan). Sphincterotomy was done before stent insertion in all cases. The length of the SEMS was determined by the primary endoscopist, and the distal end of the SEMS was located in the duodenum.

\section{RBO and adverse events}

We followed up all patients at least once a month and examined their clinical findings and biochemical parameters of hepatobiliary functions and inflammation, such as aspartate aminotransferase, alanine aminotransferase, alkaline phosphatase, $\gamma$ glutamyl transpeptidase, total and direct bilirubin, white blood cell count, and C-reactive protein. CT scanning or abdominal ultrasound was carried out at least once every 2 or 3 months until a patient's death. RBO and adverse events and their severity were defined according to the Tokyo Criteria 2014 [11]. RBO was defined as an occlusion or migration, and TRBO as the interval between SEMS placement and RBO or patients' death, whichever was earlier.

\section{Statistical analysis}

The Kaplan - Meier method was used to evaluate overall survival (OS), with living patients censored at the last date of follow-up (October 31, 2017). TRBO was also estimated by the KaplanMeier method, with patients who had not experienced RBO censored at the end of the study (October 31, 2017). The hazard ratios of prognostic factors for OS and TRBO were estimated by a Cox proportional hazards model, which included age, sex, clinical stage, chemotherapy, prior drainage, and stent types. Continuous variables were compared using the Mann- 
- Table 1 Characteristics of patients in the 12-mm-CSEEMS and 10-mm-FCSEMS groups.

\begin{tabular}{|c|c|c|c|}
\hline Patients' groups & 12-mm-CSEEMS group $(n=33)$ & 10-mm-FCSEMS group $(n=66)$ & $P$ value \\
\hline Age, years, median (range) & $75(61-92)$ & $71(36-95)$ & 0.200 \\
\hline \multicolumn{4}{|l|}{ Sex, n (\%) } \\
\hline - Male & $17(51.5)$ & $35(53.0)$ & 0.887 \\
\hline Length of stricture, mm, median (range) & $44(16-72)$ & $52(18-74)$ & 0.870 \\
\hline \multicolumn{3}{|l|}{ Length of stent, $\mathrm{n}(\%)$} & $0.643^{1}$ \\
\hline . $4 \mathrm{~cm}$ & $0(0)$ & $1(1.5)$ & \\
\hline . $6 \mathrm{~cm}$ & $9(27.3)$ & $20(30.3)$ & \\
\hline $.7 \mathrm{~cm}$ & $12(36.4)$ & $0(0)$ & \\
\hline $.8 \mathrm{~cm}$ & $12(36.4)$ & $45(68.2)$ & \\
\hline \multicolumn{4}{|l|}{ Etiology, n (\%) } \\
\hline - Pancreatic cancer & $27(81.8)$ & $51(77.2)$ & $0.602^{2}$ \\
\hline - Cholangiocarcinoma & $3(9.1)$ & $11(16.7)$ & \\
\hline - Colon cancer & $1(3.0)$ & $2(3.0)$ & \\
\hline - Intraductal papillary mucinous neoplasm & $1(3.0)$ & $0(0)$ & \\
\hline - Neuroendocrine tumor & $0(0)$ & $2(3.0)$ & \\
\hline - Gastric cancer & $1(3.0)$ & $0(0)$ & \\
\hline Clinical stage, n (\%) & 0.697 & & \\
\hline - II & $2(6.1)$ & $2(3.0)$ & \\
\hline . III & $6(18.2)$ & $15(22.7)$ & \\
\hline. IV & $25(75.8)$ & $49(74.2)$ & \\
\hline Chemotherapy, n (\%) & $20(60.6)$ & $38(57.6)$ & 0.943 \\
\hline Best supportive care, $\mathrm{n}(\%)$ & $10(30.3)$ & $28(42.4)$ & 0.342 \\
\hline
\end{tabular}

Whitney U test and Fisher's exact test for categorical variables. All analyses were done using StatFlex version 6.0 for windows (StatFlex, Osaka, Japan).

\section{Results}

\section{Patient characteristics, outcomes, and survival}

Clinical characteristics were not significantly different between the 12-mm-CSEEMS group and the $10-\mathrm{mm}$-FCSEMS group ( $\downarrow$ Table 1). In all 99 patients, placements of 12-mm CSEEMS and 10-mm FCSEMS were technically successful. Median OS of 12-mm-CSEEMS group was 232 days (range, 35-814 days), and 27 patients ( $81.8 \%$ ) died and six patients (18.2\%) were still alive by the end of the study ( $\vee$ Fig. 2 ).

Median OS of the 10-mm-FCSEMS-group was 169.5 days (range, 21 - 1019 days), and all 66 patients died by the end of the study ( $\triangleright$ Fig. 2). OS was tended to be significantly different between 12-mm-CSEEMS group and 10-mm-FCSEMS group ( $P$ $=0.081$, Fig. 2). Univariate Cox analysis demonstrated that risk of mortality was lower in patients with chemotherapy (HR, $0.610 ; 95 \% \mathrm{Cl}, 0.4041-0.92560 ; P=0.020)$, and it tended to be lower in patients with clinical stage II or III disease (HR, 0.647; $95 \% \mathrm{Cl}, 0.49376-1.03775 ; P=0.071)$ and in the 12-mm-CEEMS group (HR, 0. 667; $95 \% \mathrm{Cl}, 0.42377-1.04927$; $P=0.080$ ). Multivariate Cox hazard analysis demonstrated that risk of mortality was lower in the females (HR, 1.974; $95 \% \mathrm{Cl}, 1.23762-$ 3.14849; $P=0.004)$, in patients with clinical stage of II or III disease ( $\mathrm{HR}, 0.417 ; 95 \% \mathrm{Cl}, 0.24050-0.72313 ; P=0.002)$ and in the 12 -mm-CSEEMS group (HR, 0.592; $95 \% \mathrm{Cl}, 0.36340-$ $0.96495 ; P=0.044)(\triangleright$ Table 2$)$.

\section{RBO and TRBO}

In the 12-mm-CSEEMS group, RBO occurred in three patients (9.1\%) on days 132,155 and 505 by food impaction in one $(3.0 \%)$ and tumor ingrowth at the covered part of the stent in two $(6.1 \%)$ ( $\triangleright$ Table 3$)$.

In the 10 -mm-FCSEMS group, RBO occurred in 29 patients $(43.9 \%)$ by food impaction in two $(3.0 \%)$, sludge formation in 
Number at risk

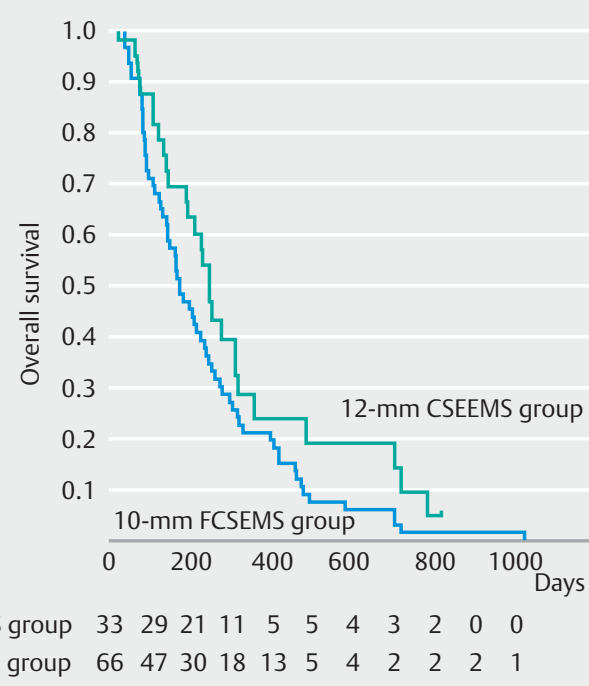

Fig. 2 Overall survival in the 12-mm-CSEEMS group and 10-mmFCSEMS group tended to be significantly different by Kaplan-Meier analysis (log rank, $P=0.0809$ ). Median OS in the 12 -mm-CSEEMS group was 232 days and median OS in the $10-\mathrm{mm}$-FCSEMS group was 169.5 days.

$13(19.7 \%)$, tumor ingrowth in one (1.5\%), tumor overgrowth in five $(7.6 \%)$, kinking in one (1.5\%), distal migration in three (4.5\%) and proximal migration in four (6.1\%) (> Table 3 ). TRBO in the 12-mm-CSEEMS group was significantly longer than that in the $10-\mathrm{mm}-\mathrm{FCSEMS}$ group (log rank, $P=0.001$ ). Median TRBO in the 12-mm-CSEEMS group was 232 days and median TRBO in the $10-\mathrm{mm}-$ FCSEMS group was 139.5 days ( Fig. 3).

Univariate Cox analysis ( $\triangleright$ Table 4) demonstrated that risk of RBO was significantly lower in the 12 -mm-CSEEMS group (HR, 0.449 ; $95 \% \mathrm{Cl}, 0.27967-0.72215 ; P=0.001)$ than in $10-\mathrm{mm}-$ FCSEMS group and chemotherapy also decreased risk of RBO (HR, 0.429; $95 \% \mathrm{Cl}, 0.27665-0.66392 ; P<0.001$ ).

Multivariate Cox hazard analysis also demonstrated that risk of RBO was significantly lower in the 12-mm-CSEEMS group than in the 10-mm-FCSEMS group (HR, 0.458; $95 \% \mathrm{Cl}$,

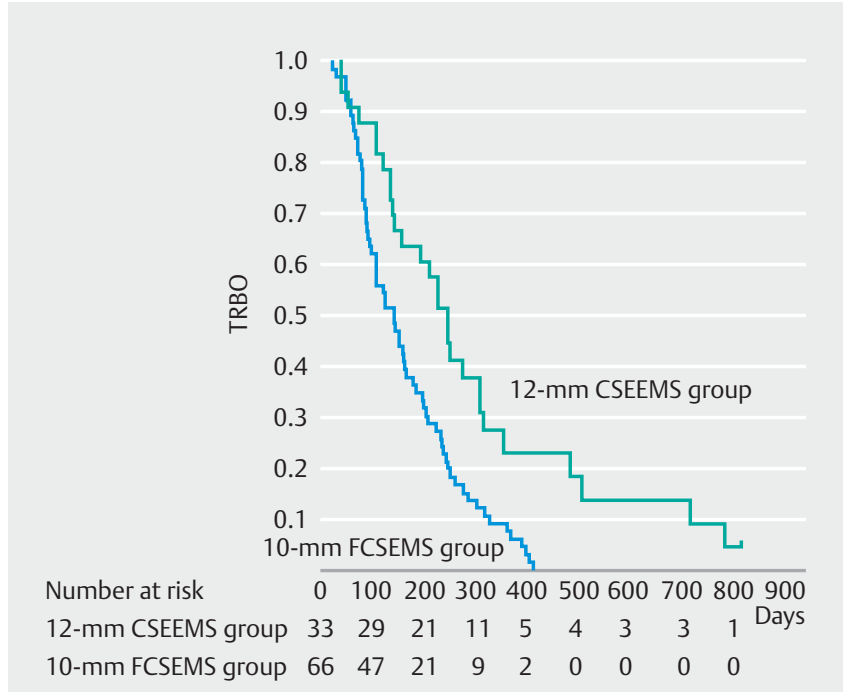

- Fig. 3 Cumulative TRBO was significantly longer in the $12-\mathrm{mm}$ CSEEMS group than in the 10-mm-FCSEMS group by Kaplan-Meier analysis (log rank, $P=0.0012$ ). Median TRBO in the 12 -mm-CSEEMS group was 232 days and median TRBO in the 10-mm-FCSEMS group was 139.5 days.

$0.28395-0.73744 ; P=0.001)$ and chemotherapy decreased risk of RBO (HR, 0.453; $95 \% \mathrm{Cl}, 0.27791-0.73974 ; P=0.002)$.

\section{Early adverse events ( $\leq 30$ days)}

In the 12-mm-CSEEMS group, there were no cases of postendoscopic retrograde cholangiopancreatography pancreatitis (PEP), while one patient (3.0\%) experienced abdominal pain on day 1 and one patient (3.0\%) experienced non-occlusion cholangitis on Day 27 ( $>$ Table 3 ). In the 10-mm-FCSEMS group, cholecystitis occurred in one patient on Day 7 (1.6\%), PEP occurred in three patients (4.5\%) and hyperamylasemia in one (1.6\%) ( $\triangleright$ Table 3$)$.There were no bleeding events in either of the two groups.

- Table 2 Univariate and multivariate Cox hazard analyses of OS.

\begin{tabular}{|c|c|c|c|c|c|c|}
\hline \multirow[t]{2}{*}{ Variables } & \multicolumn{3}{|c|}{ Univariate analysis } & \multicolumn{3}{|c|}{ Multivariate analysis } \\
\hline & Hazard ratio & $95 \% \mathrm{Cl}$ & $P$ value & Hazard ratio & $95 \% \mathrm{Cl}$ & $P$ value \\
\hline Age (years) & 1.009 & $0.99109-1.02624$ & 0.340 & 1.0103 & $0.98964-1.0318$ & 0.330 \\
\hline Sex, male & 1.512 & $0.99728-2.29347$ & 0.052 & 1.974 & $1.23762-3.14849$ & 0.004 \\
\hline Primary disease (pancreatic cancer) & 1.092 & $1.09198-1.85604$ & 0.745 & & & \\
\hline Clinical stage (II and III) & 0.647 & $0.40376-103775$ & 0.071 & 0.417 & $0.24050-0.72313$ & 0.002 \\
\hline Chemotherapy & 0.610 & $0.4041-0.92560$ & 0.020 & 0.744 & $0.45211-1.22419$ & 0.245 \\
\hline 12-mm CSEEMS & 0.667 & $0.42377-1.04927$ & 0.080 & 0.592 & $0.36340-0.96495$ & 0.044 \\
\hline
\end{tabular}

OS, overall survival; CSEEMS, covered, self-expandable end bare metal stent 
- Table 3 Recurrent biliary obstruction and adverse events in 12-mm-CSEEMS and 10-mm-FCSEMS groups.

\begin{tabular}{|c|c|c|c|c|c|}
\hline \multirow[b]{2}{*}{ Recurrent biliary obstruction, $\mathrm{n}(\%)$} & \multicolumn{2}{|c|}{ 12-mm-CSEEMS group $(n=33)$} & \multicolumn{2}{|c|}{ 10-mm-CSEMS group $(n=66)$} & \multirow{2}{*}{$\begin{array}{l}\boldsymbol{P} \text { value } \\
0.001\end{array}$} \\
\hline & $3(9.1)$ & & $29(43.9)$ & & \\
\hline Occlusion, n (\%) & $3(9.1)$ & & $22(33.3)$ & & 0.009 \\
\hline - Food impaction & $1(3.0)$ & & $2(3.0)$ & & 1.000 \\
\hline - Sludge & 0 & & $13(19.7)$ & & 0.009 \\
\hline - Ingrowth & $2(6.1)$ & & $1(1.5)$ & & 0.549 \\
\hline - Overgrowth & 0 & & $5(7.6)$ & & 0.166 \\
\hline - Hemobilia & 0 & & 0 & & 1.000 \\
\hline - Kinking & 0 & & $1(1.5)$ & & 1.000 \\
\hline Migration, n (\%) & 0 & & $7(10.6)$ & & 0.092 \\
\hline - Distal migration & 0 & & $3(4.5)$ & & 0.549 \\
\hline - Proximal migration & 0 & & $4(6.1)$ & & 0.298 \\
\hline \multicolumn{6}{|l|}{ Adverse events, $n$ (\%) } \\
\hline Early adverse events ( $\leq 30$ days) & $2(6.1)$ & & $5(7.6)$ & & 1.000 \\
\hline - Cholecystitis & 0 & & $1(1.6)$ & on day 7 & 1.000 \\
\hline - Pancreatitis & 0 & & $3(4.5)$ & on day 1 & 0.298 \\
\hline - Hyperamylasemia & 0 & & $1(1.6)$ & on day 1 & 1.000 \\
\hline - Abdominal pain & $1(3.0)$ & on day 1 & 0 & 0.333 & \\
\hline - Non-occlusion cholangitis (moderate) & $1(3.0)$ & on day 27 & 0 & & 0.333 \\
\hline Late adverse events ( $\geq 31$ days) & $3(9.1)$ & & $8(12.1)$ & & 0.747 \\
\hline - Cholecystitis (moderate) & $1(3.0)$ & on day 77 & $1(1.6)$ & on day 32 & 1.000 \\
\hline - Non-occlusion cholangitis (moderate) & $2(6.1)$ & on days 116 and 151 & $7(10.6)$ & $\begin{array}{l}\text { on days } 82,108,116 \text {, } \\
132,146,172 \text { and } 196\end{array}$ & 0.714 \\
\hline
\end{tabular}

- Table 4 Univariate and multivariate Cox hazard analyses of TRBO.

\begin{tabular}{|l|l|l|l|l|l|}
\hline Variables & \multicolumn{2}{|l|}{ Univariate analysis } & \multicolumn{3}{l|}{ Multivariate analysis } \\
\hline & Hazard ratio & $\mathbf{9 5 \%} \mathbf{C l}$ & $\mathbf{P}$ value & Hazard ratio & $\mathbf{9 5 \%} \mathbf{C l}$ \\
\hline Age (years) & 1.012 & $0.99379-1.03151$ & 0.192 & 1.000 & $0.98209-1.01913$ \\
\hline Sex, male & 1.183 & $0.85001-1.93025$ & 0.2367 & 1.189 & $0.78224-1.80824$ \\
\hline $\begin{array}{l}\text { Primary disease } \\
\text { (pancreatic cancer) }\end{array}$ & 0.880 & $0.52295-1.48068$ & 0.6300 & & 0.891 \\
\hline Clinical stage (II and III) & 0.711 & $0.44559-1.13394$ & 0.1520 & & \\
\hline Chemotherapy & 0.429 & $0.27665-0.66392$ & 0.0001 & 0.453 & $0.27791-0.73974$ \\
\hline 12-mm CSEEMS & 0.449 & $0.27967-0.72215$ & 0.0009 & 0.458 & 0.002 \\
\hline TRBO, time to recurrent biliary obstruction; CSEEMS, covered, self-expandable end bare metal stent & \\
\hline
\end{tabular}




\section{Late adverse events ( $\geq 31$ days)}

In the 12-mm-CSEEMS-group, acute cholecystitis occurred in one patient on Day 77 (3.0\%) and non-occlusion moderate cholangitis occurred in two patients (6.1\%) on Days 116 and 151 ( $\triangleright$ Table 3 ). In the 10-mm-FCSEMS group, acute cholecystitis occurred in one on Day 32 (1.6\%) and non-occlusion cholangitis occurred in seven patients (10.6\%) ( $\triangleright$ Table 3$)$.

\section{Discussion}

Endoscopic drainage of the common bile duct using SEMS is an effective and widely performed treatment for unresectable malignant biliary obstruction. For patients with unresectable tumors, SEMS placement maintains biliary flow, relieves jaundice, improves quality of life, and facilitates delivery of consecutive chemotherapy.

In this study, 12-mm CSEEMS showed a longer TRBO compared with $10-\mathrm{mm}$ FCSEMS. TRBO was significantly longer in the $12-\mathrm{mm}$-CSEEMS group than in the 10 -mm-FCSEMS group (log rank, $P=0.001)$ and both univariate ( $\mathrm{HR}, 0.449 ; 95 \% \mathrm{Cl}$, 0.27967-0.72215; $P=0.001)$ and multivariate (HR, 0.458; $95 \% \mathrm{Cl}, 0.28395-0.73744 ; P=0.001)$ Cox hazard analysis found that $12-\mathrm{mm}$ CSEEMS was associated with a significantly lower risk of RBO. In the 12-mm-CSEEMS group, median TRBO was 232 days and was equal to median OS, on the other hand, median TRBO was 139.5 days, and the median OS was 169.5 days in 10-mm-FCSEMS group.

Because the time of treatment differed between the two groups, patients with pancreatic cancer in the 12-mm-CSEEMS group were treated with newly developed chemotherapy, while those in the 10-mm-FCSEMS received an older chemotherapy regimen $[12,13]$. In the 10 -mm FCSEMS-group, 30 out of 38 patients (78.9\%) undergoing chemotherapy had pancreatic cancer, of whom FOLFILINOX was done in three cases, GnP in one, GEM in 24 and S-1 in two cases. On the other hand, in the 12 -mm-CSEEMS group, 18 out of 20 patients (90\%) undergoing chemotherapy had pancreatic cancer, of whom FOLFILINOX was done in one case, GnP in 14, GEM in tw and S-1 in one patient. Thus, tumors in the 12-mm-CSEEMS group may have been more effectively controlled than those in the 10-mm-FCSEMS group. The longer TRBO in the 12-mm-CSEEMS group may be affected by the difference in chemotherapy regimen. Thus, a further prospective study is needed to compare TRBO between the two groups.

A meta-analysis of RCT reported better stent patency with CSEMS than with USEMS [6]. It also reported that risk of migration was greater with CSEMS and that there were no differences between CSEMS and USEMS in occurrence of adverse events such as pancreatitis or cholecystitis. Other meta-analyses of CSEMS and USEMS found no benefit for CSEMS [14-16]. In our study, stent patency rate at 6 months was $91.7 \%$ with $12-\mathrm{mm}$ CSEEMS, and we did not experience stent migration. With the 10-mm-diameter partially-covering SEMS, stent migration occurred in $7.8 \%$ of patients over 1 year in the WATCH study [17]. In that study, 10-mm FCSEMS migrated in seven patients (10.6\%) during the observation period of 12 to 410 days. We believe that the 12-mm CSEEMS proximally bare is effective for prevention of migration and the larger-caliber style appears to be effective for preventing occlusion.

Compared with USEMS, FCSEMS has the possibility of removal. CSEEMSs were not removed because the proximal end bare might injure the bile duct. In two RBO cases caused by tumor in-growth in the 12-mm-CSEEMS group, stent-in-stent placement was performed and in one RBO case caused by food impaction, cleaning was performed. In these three cases, no further RBO was experienced.

In the 12-mm-CSEEMS group, two patients experienced tumor ingrowth $(2 / 33,6.1 \%)$ at the covered portion of the stent. Therefore, an improvement in the membrane may be needed.

The low incidence of non-occlusion cholangitis (3/33, 9.1\%) associated with 12-mm CSEEMS was satisfactory and similar to the $5.7 \%$ associated with $10-\mathrm{mm}$ CSEMS reported in the WATCH study [17].

Pancreatitis did not occur in any patients with 12-mm CSEEMS. Kawakubo et al. [18] reported that a high AF and primary diseases other than pancreatic cancer were risk factors for pancreatitis after SEMS placement. Pancreatitis occurred in three patients with $10-\mathrm{mm}$ FCSEMS (3/66, $4.5 \%$ ), all of whom were treated conservatively. This result was consistent with occurrence of pancreatitis of $5.9 \%$ associated with CSEMSs [18]. We experienced one case of abdominal pain in a patient with a bile duct diameter $<7 \mathrm{~mm}$ and a $12-\mathrm{mm}$ CSEEMS. Pain was controlled with medication and disappeared after 1 day.

One patient $(1 / 33,3.0 \%)$ in the 12 -mm-CSEEMS group experienced moderate cholecystitis as defined by the Tokyo 2014 criteria [11]. That patient had gallstones, and the tumor involved the orifice of the cystic duct. That patient gradually improved with temporary percutaneous intervention. Similar findings have been previously reported $[19,20]$ and in this series, one patient with 10-mm FCSEMS and tumor involvement of the orifice of the cystic duct and a gallstone experienced cholecystitis $(1 / 66,1.6 \%)$ as a late adverse event.

Limitations of this study include a small non-randomized patient sample. However, because there have been no reports on this $12-\mathrm{mm}$ CSEEMS, we think that it is meaningful to report this study which demonstrates the superiority of this device.

\section{Conclusion}

The 12-mm CSEEMS showed a longer TRBO compared with the widely used 10-mm FCSEMS, with a similar incidence of adverse events. Therefore, this stent may be safe and effective for managing malignant distal biliary obstruction. A randomized controlled trial comparing the novel 12-mm CSEEMS with a conventional $10-\mathrm{mm}$ CSEMS is planned to assess possible superiority.

\section{Competing interests}

None 


\section{References}

[1] Irving JD, Adam A, Dick R et al. Gianturco expandable metallic biliary stents: results of a European clinical trial. Radiology 1989; 172: 321 326

[2] Lammer ], Klein GE, Kleinert R et al. Obstructive jaundice: use of expandable metal endoprosthesis for biliary drainage. Work in progress. Radiology 1990; 177: 789-792

[3] Davids PH, Groen AK, Rauws EA et al. Randomised trial of self-expanding metal stents versus polyethylene stents for distal malignant biliary obstruction. Lancet 1992; 340: 1488-1492

[4] Hoepffner N, Foerster EC, Hogemann B et al. Long-term experience in Wallstent therapy for malignant choledochal stenosis. Endoscopy 1994; 26: 597-602

[5] Isayama H, Komatsu Y, Tsujino T et al. A prospective randomised study of "covered" versus "uncovered" diamond stents for the management of distal malignant biliary obstruction. Gut 2004; 53: 729 734

[6] Saleem A, Leggett CL, Murad MH et al. Meta-analysis of randomized trials comparing the patency of covered and uncovered self-expandable metal stents for palliation of distal malignant bile duct obstruction. Gastrointest Endosc 2011; 74: 321-327.e321-323

[7] Kitano M, Yamashita Y, Tanaka K et al. Covered self-expandable metal stents with an anti-migration system improve patency duration without increased complications compared with uncovered stents for distal biliary obstruction caused by pancreatic carcinoma: a randomized multicenter trial. Am J Gastroenterol 2013; 108: 1713-1722

[8] Almadi MA, Barkun AN, Martel M. No benefit of covered vs uncovered self-expandable metal stents in patients with malignant distal biliary obstruction: a meta-analysis. Clin Gastroenterol Hepatol 2013; 11: $27-37 . e 21$

[9] Mukai T, Yasuda I, Isayama $\mathrm{H}$ et al. Pilot study of a novel, large-bore, fully covered self-expandable metallic stent for unresectable distal biliary malignancies. Dig Endosc 2016; 28: 671-679
[10] Isayama H, Nakai Y, Toyokawa Y et al. Measurement of radial and axial forces of biliary self-expandable metallic stents. Gastrointest Endosc 2009; 70: $37-44$

[11] Isayama H, Hamada T, Yasuda I et al. TOKYO criteria 2014 for transpapillary biliary stenting. Dig Endosc 2015; 27: 259-264

[12] Ramanathan RK, Goldstein D, Korn RL et al. Positron emission tomography response evaluation from a randomized phase III trial of weekly nab-paclitaxel plus gemcitabine versus gemcitabine alone for patients with metastatic adenocarcinoma of the pancreas. Ann Oncol 2016; 27: $648-653$

[13] Conroy T, Desseigne F, Ychou M et al. FOLFIRINOX versus gemcitabine for metastatic pancreatic cancer. N Engl J Med 2011; 364: 1817 - 1825

[14] Chen MY, Lin JW, Zhu HP et al. Covered Stents versus uncovered stents for unresectable malignant biliary strictures: a meta-analysis. BioMed Res Int 2016; 2016: 6408067

[15] Yang Z, Wu Q, Wang F et al. A systematic review and meta-analysis of randomized trials and prospective studies comparing covered and bare self-expandable metal stents for the treatment of malignant obstruction in the digestive tract. Int J Med Sci 2013; 10: 825-835

[16] Li J, Li T, Sun P et al. Covered versus uncovered self-expandable metal stents for managing malignant distal biliary obstruction: a meta-analysis. PloS One 2016; 11: e0149066

[17] Isayama H, Mukai T, Itoi T et al. Comparison of partially covered nitinol stents with partially covered stainless stents as a historical control in a multicenter study of distal malignant biliary obstruction: the WATCH study. Gastrointest Endosc 2012; 76: 84-92

[18] Kawakubo K, Isayama H, Nakai Y et al. Risk factors for pancreatitis following transpapillary self-expandable metal stent placement. Surg Endosc 2012; 26: $771-776$

[19] Isayama H, Kawabe T, Nakai Y et al. Cholecystitis after metallic stent placement in patients with malignant distal biliary obstruction. Clin Gastroenterol Hepatol 2006; 4: 1148-1153

[20] Suk KT, Kim HS, Kim JW et al. Risk factors for cholecystitis after metal stent placement in malignant biliary obstruction. Gastrointest Endosc 2006; 64: $522-529$ 